\title{
Effects of Bifidobacterium longum BB536 on lipid profile and histopathological changes in hypercholesterolaemic rats
}

\begin{abstract}
The present study investigated the effects of Bifidobacterium longum BB536 on lipid profile, liver and kidney function, and body fat in hypercholesterolaemic rats. 40 Sprague-Dawley rats were randomly divided into five groups. The negative control group received a standard diet. The positive control group received a cholesterol-enriched diet, whereas the intervention groups received a cholesterol-enriched diet supplemented with B. longum BB536 alone or in combination with inulin or Mangifera pajang fibrous polysaccharides. After 8 weeks, plasma lipids, and liver and kidney function were tested. Intake of the cholesterol-enriched diet increased total cholesterol, alanine aminotransferase, gamma-glutamyl transferase, creatinine, urea, liver weight, adipose tissue weight, liver lipid deposition and adipocyte size. B. longum BB536 supplementation significantly reduced total cholesterol, liver lipid deposition and adipocyte size, and positively affected liver and kidney function. These effects were significantly increased in the presence of inulin and M. pajang fibrous polysaccharides.
\end{abstract}

Keyword: Bifidobacteria; Histology; Hypocholesterolaemic rats; Lipid profile 